\title{
Review of: "Current biological approaches for management of crucifer pests"
}

\section{Dr. Muhammad Hafeez}

Potential competing interests: The author(s) declared that no potential competing interests exist.

good 